\title{
Efecto De Di Ferentes Intensidades De Poda De Formación En El Crecimiento Inicial De Melina (Gmelina Arborea Roxb.)
}

\author{
Norma Ximena Lara Vasconez, (Ing. For. Msc.), \\ Eduardo Cevallos (Ing. Agr.Msc), \\ Santiago Almeida (Ing. For.), \\ Sulema Lara (Ing. Ecot. Msc.)
}

Universidad / Escuela Superior Politécnica de Chimborazo

doi: 10.19044/esj.2016.v12n36p95 URL:http://dx.doi.org/10.19044/esj.2016.v12n36p95

\begin{abstract}
This research aims to evaluate the effect of different intensities of formation pruning in the initial development of the Melina (Gmelina arborea Roxb.) in the Pedro Vicente Maldonado canton located in the Pichincha Province. An established Melina (beech wood) plantation, with three different intensities of pruning were used; the experimental design utilized 3 pruning treatments on randomized complete blocks and the control plot, 4 repetitions with a total of 16 experimental units, the investigation began at 3 months after planting and finished when they were 8 months old. The results being that the best diameter was obtained with the pruning of $60 \%$ of the tree, the height didn't have any significant statistical difference between treatments, the best straightness was obtained with 50\% and $70 \%$ pruning, the highest number of branches pruned were pruned $70 \%$, the control tree has the biggest number of broken off branches. The conclusion being that the best increments in diameters were obtained by the control plot; however, this pruning treatment is rejected because the purpose is to obtain wood free of knots. Without taking into consideration the control plot, the best diameters were obtained by pruning $60 \%$, but the form of the tree trunk presents a slight curve in relationship to the intensity of pruning $50 \%$ and $70 \%$, where the trees are straighter but with smaller diameters. The formation pruning doesn't influence the height growth of the plant, but it is positive in the increment of the diameter and the straightness. The lack of pruning in the Gmelina causes the breaking off of branches causing damage to the trunk of the tree.
\end{abstract}

Keywords: Gmelina tree, silviculture, prunning, initial growing. 


\section{Resumen}

La presente investigación propone evaluar el efecto que producen distintas intensidades de poda de formación en el desarrollo inicial de Melina (Gmelina arborea Roxb.) en el Cantón Pedro Vicente Maldonado provincia de Pichincha, Ecuador. Una plantación de melina ya establecida, con tres diferentes intensidades de poda fue utilizada; el diseño experimental empleado fue de bloques completos al azar con 3 tratamientos más el testigo y 4 repeticiones con un total de 16 unidades experimentales, la investigación empezó a los 3 meses de establecida la plantación y terminó a los 8 meses de edad. Dando como resultado que el mejor diámetro obtuvo la poda al $60 \%$, la altura no tuvo diferencia estadística significativa entre tratamientos, la mejor rectitud de fuste obtuvo el tratamiento con poda al $50 \%$ y $70 \%$, el mayor número de ramas podadas tuvo la poda al 70\%, el testigo tiene el mayor número de ramas desgajadas. Se concluye que los mejores incrementos en diámetros fueron alcanzados por el testigo o control, sin embargo, este tratamiento es desestimado ya que el propósito es obtener madera libre de nudos. Con la poda del $60 \%$ se obtienen los mejores diámetros después del testigo, pero la forma del fuste del árbol presenta una curva leve con relación a la intensidad de poda del 50\% y $70 \%$ donde los árboles son más rectos pero con diámetros menores. Las podas de formación no influyen en el crecimiento en altura de la planta, pero si en el incremento del diámetro y la rectitud del fuste. La falta de poda en melina ocasiona el desgaje de las ramas provocando daño al fuste del árbol.

Palabras clave: Melina, silvicultura, poda, crecimiento inicial.

\section{Introducción}

En una significativa mayoría de proyectos, tanto privados como estatales, erróneamente se ha visto y tratado la plantación de árboles como una actividad simple, en el sentido de que luego del establecimiento, los responsables de la actividad emprenden muy pocas acciones de manejo y sólo esperan un tiempo para cosechar la madera y recuperar la inversión; óptica que ha llevado, al fracasado de múltiples inversiones de reforestación (TRUJILLO, E. 2007).

La misma consecuencia ha tenido el hecho de que no se planifiquen las inversiones propias del flujo de caja durante la vida de la plantación, o que cuando se requieren acciones de manejo que garanticen la calidad de la madera, estas no se ejecuten por falta de recursos, lo que invariablemente afecta no sólo la calidad sino hasta la permanencia misma de la plantación; en este sentido, es desconocido que los proyectos de reforestación deben tener 
seguridad, antes del establecimiento, de las inversiones a lo largo de la vida del proyecto.

También ocurre que las inversiones para el manejo, las determina un proceso administrativo que asigna recursos sujeto a sus propios condicionamientos y no a los condicionamientos y requerimientos técnicos de la plantación, o que no emprende actividades en el momento que se requieren para evitar daños en la estructura de la plantación o la calidad de la madera.

La poda es una de las prácticas básicas pero determinantes en el óptimo desarrollo de las plantaciones, una acción de manejo que influye decididamente en el valor de la madera y por tanto en la rentabilidad del proyecto (TRUJILLO, E. 2007).

Para conocer en detalle la adaptación de una especie a determinada localidad, se deben evaluar distintos factores ambientales y dasométricos como son el suelo, la precipitación, sobrevivencia, crecimientos en follaje, diámetro, altura, sanidad y vigor. Adicional al conocimiento de la adaptación de las especies, se debe tomar decisiones para la realización de podas y aclareos, los cuales tendrán influencia en la cantidad y calidad de madera esperada en el futuro. Las evaluaciones periódicas proporcionan el detalle necesario para realizar silvicultura de plantaciones, como son las podas, aclareos, entre otras., (TORRES y MAGAÑA, 2001).

El protocolo de poda, como en todas las acciones de manejo de plantaciones forestales, no se puede generalizar; en algunos casos, el crecimiento exuberante de algunas especies como la Acacia mangium en la Orinoquia, por ejemplo, exige la realización de podas tempranas, y aunque la pérdida de área foliar disminuye el crecimiento, este compensa con la rapidez de su crecimiento y con la ventaja de una mejora en la calidad de su madera desde un principio. (TRUJILLO, E. 2007)

Por lo antes mencionado se planteó la presente investigación con la finalidad de evaluar el efecto de diferentes intensidades de poda de formación en el crecimiento inicial de melina en el cantón Pedro Vicente Maldonado provincia de Pichincha, en búsqueda de alternativas productivas, económicas para el desarrollo de esta especie, se planteó el siguiente objetivo general: Evaluar el efecto que producen distintas intensidades de poda de formación en el desarrollo inicial de melina en el Cantón Pedro Vicente Maldonado Provincia de Pichincha.

\section{Características del lugar}

La temperatura anual oscila entre $24-26^{\circ} \mathrm{C}$, la precipitación anuales de $2000-4000 \mathrm{~mm}$, la evapotranspiración es de $0.08 \mathrm{~mm}$, velocidad del viento es de $1.2 \mathrm{Km} / \mathrm{h}$. El suelo se clasifica como Latosol, arcillo-rojizos. Según HOLDRIDGE (1992) la formación ecológica dominante corresponde a 
Bosque Húmedo Tropical (bh-T). La topografía tiene zonas continuas y discontinuas, pendientes regulares y onduladas.

\section{Metodología}

Para la presente investigación se utilizó la especie forestal melina con la combinación de tres diferentes intensidades de poda. Para el campo experimental se utilizaron parcelas de forma rectangular. Cada unidad experimental consta de 28 plantas de las cuales solo 10 fueron seleccionadas para las evaluaciones. La superficie total es de $7168 \mathrm{~m}^{2}$, la parcela experimental de $448 \mathrm{~m}^{2}$, la parcela neta de $160 \mathrm{~m}^{2}$, el número de unidades experimentales es de 16, con 160 plantas a evaluarse. Los tratamientos estuvieron constituidos por la determinación de la intensidad de poda de formación más adecuada para melina en la zona de estudio, la cual fue conformada por 3 tratamientos más el testigo y cuatro repeticiones. Los tratamientos a evaluar son el T0 o testigo, T1 al cual se le aplicó una poda al $50 \%$, T2 al cual se le aplicó una poda al 60\%, T3 al cual se le aplicó una poda al $70 \%$.Cadea uno de los tratamientos fue realizado con cuatro repeticiones. El diseño que se utilizó fue de Bloques Completos al Azar (BCA), en donde se establecieron 16 unidades experimentales.

\section{Análisis estadístico}

En el cuadro 1, se presenta el esquema del análisis de varianza que se utilizó en el ensayo.

Tabla 1. Análisis de varianza (ADEVA)

\begin{tabular}{|c|c|c|}
\hline Fuente de Variación & Fórmula & $\mathrm{G} \mathrm{L}$ \\
\hline Bloques & $\mathrm{r}-1$ & 3 \\
Tratamientos & $\mathrm{a}-1$ & 3 \\
Error & $(\mathrm{a}-1)(\mathrm{r}-1)$ & 5 \\
Total & $\mathrm{a} * \mathrm{n}-1$ & 11 \\
\hline
\end{tabular}

Se determinó el coeficiente de variación (CV) expresado en porcentaje. Para la separación de medias se aplicó la prueba de Tukey al $5 \%$.

Esta investigación dio inicio en el rodal DB32 el cual tenía 3 meses de establecida la plantación y se encontraba apto para la poda de formación I.

Previo a la primera poda se registraron datos de diámetro, altura, rectitud del fuste y número de plantas bifurcadas, esto para tener una base de información y saber en qué estado se encontraba la plantación antes de iniciada la investigación. El mismo día posterior a esta medición se realizó la poda de formación I, la cual consistió en podar ramas basales y corte de ápices laterales, siempre tratando de dejar equilibrada a la planta en lo que se refiere a cantidad de ramas de lado y lado. Además luego de haber realizado la poda se registraron datos del número de ramas podadas. 
Los siguientes dos meses después de realizada la poda de formación I, se registraron datos de diámetros, alturas y plantas bifurcadas. A los 6 meses de establecida la plantación se realizó la poda de formación II, aquí al igual que en la poda de formación I se podó ramas basales y corta de ápices laterales. Los datos de rectitud del fuste se registraron previo a la poda de formación I y dos meses después de la poda de formación II. Los datos de desgaje, es decir desgarre de ramas por sobrepeso y colapso es decir, todas la ramas se desgarran pudiendo quebrar el fuste; solo se registraron en los testigos.

\section{Evaluación del crecimiento inicial}

Diámetro.- Las 3 primeras mediciones del diámetro fueron registradas con el calibrador (pie de rey) a la altura del DAC (Diámetro altura del cuello), para esto, a $10 \mathrm{~cm}$ de altura desde el suelo se realizó en el tallo un anillo pintado de color rojo, para poder tomar las medidas en el mismo lugar los siguientes meses. Las 3 últimas mediciones se las realizó con la utilización de una forcípula dado que las plantas ganaron mayor altura (6 meses de edad; altura promedio 2 metros) por lo que fue necesario tomar el DAP (Diámetro a la altura del pecho), previo a esto se pintó un anillo color rojo a la altura de 130 $\mathrm{cm}$ desde la base de la planta.

Altura.- En la base de cada planta se enterró una estaca dejándola al nivel del suelo y luego se procedió a pintar de color rojo la parte superficial para poderla ubicar con mayor facilidad, esta estaca nos sirvió para poder asentar la regleta y así poder tomar las alturas mes a mes desde una misma base. Las alturas totales de cada planta fueron registradas mediante la utilización de una regleta graduada, las mismas fueron medidas desde la base de la planta hasta la punta o ápice principal. Este registro se lo realizó en 6 ocasiones.

Rectitud del fuste.- Para evaluar la rectitud del fuste se dividió al árbol en 2 tramos:

Tramo A= Es la parte media baja del fuste, es decir, desde la mitad de la altura total del árbol hacia la base del mismo y se procedió a evaluar con una escala de 1 a 5 por observación visual. Tramo $\mathrm{B}=$ Parte media alta del fuste y su evaluación fue similar a la anterior.

Tabla 2. Escala para la valoración de la rectitud del fuste

\begin{tabular}{|c|c|}
\hline Forma del fuste & Rangos \\
Recto & (Suma de tramo A y B) \\
\hline Curva leve & $8,1-10$ puntos \\
Curva pronunciada & $2,1-4$ puntos \\
Doble curva & $1-2$ puntos \\
\hline
\end{tabular}

Fuente: HERRERA, B. (2013) 
Siendo un árbol perfecto el que en la suma alcance el valor más cercano a 10 puntos y árbol defectuoso aquel que tenga calificaciones menores, para el fuste calculado. Esta evaluación se la realizó antes de la poda de formación I y dos meses después de la poda de formación II, es decir antes de iniciada la investigación y al finalizar la misma.

Plantas bifurcadas.- En cada medición se realizó el conteo de plantas que poseían doble ápice para de esta manera determinar la cantidad de plantas que pierden dominancia apical.

Ramas podadas.- Posterior a cada poda se contabilizó el número de ramas podadas, para con esto poder determinar si las diferentes intensidades de poda influyen en el comportamiento de la planta en cuanto al número de ramas se refiere.

Desgajes.- Esta evaluación se realizó únicamente en los testigos (T0), para esto se procedió a realizar el conteo de todas ramas desgajadas que tenían las diferentes plantas para con esto poder comprobar si la poda influye o no en el desgaje de las mismas.

Determinación de la intensidad de poda más adecuada.- Con los parámetros evaluados y la información recopilada se realizó el estudio estadístico para lo cual se ejecutó el análisis de varianza, se determinó el coeficiente de variación y se utilizó la prueba de Tukey al 5\% para la separación de medias.

\section{Resultados y discusión}

El crecimiento inicial en plantas de melina con tres intensidades de poda de formación muestra los siguientes resultados en:

\section{Crecimiento en diámetro}

El análisis de varianza para el diámetro inicial no presentó diferencia estadística significativa entre tratamientos. En promedio el diámetro inicial fue $2.22 \mathrm{~cm}$. El coeficiente de variación fue $18.33 \%$. El análisis de varianza para el diámetro en la segunda lectura, presentó diferencia estadística significativa entre tratamientos. En promedio el diámetro en la segunda lectura fue $3.61 \mathrm{~cm}$. El coeficiente de variación fue $13.54 \%$.

En la prueba de Tukey al 5\% para el diámetro en la segunda lectura, presentó 3 rangos; el testigo (T0), se ubicó en el rango " $\mathrm{A}$ " con un valor de $4.44 \mathrm{~cm}$., mientras que la poda $70 \%$ (T3) y poda $50 \%$ (T1) se ubicaron en el rango "B" con valores de 3.31 y $3.19 \mathrm{~cm}$., cada uno de ellos respectivamente, la otra poda se ubicó en un rango. El análisis de varianza para el diámetro en la tercera lectura, presentó diferencia estadística significativa entre tratamientos.

En promedio el diámetro en la tercera lectura fue $5.44 \mathrm{~cm}$. El coeficiente de variación fue $11.85 \%$. En la prueba de Tukey al $5 \%$ para el 
diámetro en la tercera lectura, presentó 3 rangos; el testigo (T0) se ubicó en el rango "A" con un valor de $6.39 \mathrm{~cm}$., mientras que la poda $50 \%$ (T1) se ubicó en el rango "C" con un valor de $4.78 \mathrm{~cm}$., los otros tratamientos se ubicaron en un rango intermedio.

La especie melina es una especie de rápido crecimiento, su diámetro aumenta aceleradamente en los primero años hasta presentar más de $80 \mathrm{~cm}$ de diámetro; según Rojas, et al. (2004) el diámetro en las primeras etapas de crecimiento de la melina alcanza los $7 \mathrm{~cm}$ siendo este valor creciente; lo que concuerda con lo expuesto en la presente investigación en la cual se ha obtenido un valor de $6.39 \mathrm{~cm}$.

El análisis de varianza para el diámetro en la cuarta lectura, no presentó diferencia estadística significativa entre tratamientos, en promedio el diámetro en la cuarta lectura fue $3.35 \mathrm{~cm}$. y el coeficiente de variación $21.46 \%$.

El análisis de varianza para el diámetro en la quinta lectura, no presentó diferencia estadística significativa entre tratamientos. En promedio el diámetro en la quinta lectura fue $6.59 \mathrm{~cm}$. y el coeficiente de variación 10.74 $\%$.

El análisis de varianza para el diámetro en la sexta lectura no presentó diferencia estadística significativa entre tratamientos. En promedio el diámetro en la sexta lectura fue $6.80 \mathrm{~cm}$., y el coeficiente de variación fue 9.76 $\%$.

Según lo expuesto por VALLEJO, 1996. La melina alcanza un diámetro de $4.63 \mathrm{~cm}$ a los 12 meses, valor con el cual tiene una clasificación excelente; realizando una relación para los 8 meses, tiempo en el cual se realizó la última medición se obtiene un valor de $3.09 \mathrm{~cm}$ de diámetro, lo que comparado con el dato de la presente investigación en la cual se obtuvo una media de $5.47 \mathrm{~cm}$ de diámetro, valor que es mayor al expuesto por el autor con lo cual tendríamos una calificación de excelente.

Los diámetros obtenidos en las diferentes evaluaciones se muestran con valores distintos debido a que las tres primeras mediciones se realizó a 10 $\mathrm{cm}$ del suelo, posterior a esto la melina desarrolló una anomalía, denominada pata de elefante, que probablemente se debe a particularidades propias de la especie, esta se presentó a partir de los 5 meses de edad aproximadamente; mientras que en la cuarta, quinta y sexta edición se tomó el DAP.

\section{Crecimiento en altura}

El análisis de varianza para la altura de planta primera lectura, no presentó diferencia estadística significativa entre tratamientos. En promedio la altura de planta inicial fue $1.16 \mathrm{~m}$. El coeficiente de variación fue $17.34 \%$.

El análisis de varianza para la altura de planta en la segunda lectura, no presentó diferencia estadística significativa entre tratamientos. En 
promedio la altura de planta en la segunda lectura fue $1.84 \mathrm{~m}$. El coeficiente de variación fue $15.96 \%$.

El análisis de varianza para la altura de planta en la tercera lectura, no presentó diferencia estadística significativa entre tratamientos. En promedio la altura de planta en la tercera lectura fue $2.52 \mathrm{~m}$. El coeficiente de variación fue $14.86 \%$.

El análisis de varianza para la altura de planta en la cuarta lectura, no presentó diferencia estadística significativa entre tratamientos. En promedio la altura de planta en la cuarta lectura fue $3.08 \mathrm{~m}$. El coeficiente de variación fue $14.56 \%$.

El análisis de varianza para la altura de planta en la quinta lectura, no presentó diferencia estadística significativa entre tratamientos. En promedio la altura de planta en la quinta lectura fue $3.79 \mathrm{~m}$. El coeficiente de variación fue $12.91 \%$.

El análisis de varianza para la altura de planta en la sexta lectura, no presentó diferencia estadística significativa entre tratamientos. En promedio la altura de planta en la sexta lectura fue $4.38 \mathrm{~m}$. El coeficiente de variación fue $11.91 \%$.

Según Obregón, (2005) la principal cualidad es su acelerado crecimiento hasta los cinco o seis años de edad, ya que cuando alcanza la altura de los ejemplares adultos, su crecimiento se vuelve lento; lo que concuerda con los resultados obtenidos en la presente investigación en la cual se nota el crecimiento acelerado desde la primera lectura que es de $1.16 \mathrm{~m}$., hasta alcanzar los $4.38 \mathrm{~m}$. a los 8 meses de evaluación, valor que supera a lo expuesto por VALLEJO, 1996., el cual alcanza una altura de $3.91 \mathrm{~m}$ a los 12 meses y obtiene una calificación de excelente.

\section{Rectitud del fuste}

El análisis de varianza para el tramo medio bajo, no presentó diferencia estadística significativa entre tratamientos. En promedio el tramo medio bajo fue 3.71. El coeficiente de variación fue $9.21 \%$.

El análisis de varianza para el tramo medio bajo, no presentó diferencia estadística significativa entre tratamientos. En promedio el tramo medio bajo fue 3.78. El coeficiente de variación fue $13.01 \%$.

El tramo medio bajo presenta una media de 3.71 y 3.78 en la primera y segunda lectura respectivamente lo cual corresponde a una curva leve según la tabla de interpretación.

En el análisis de varianza para el tramo medio alto, no presentó diferencia estadística significativa entre tratamientos. En promedio el tramo medio alto fue 4.75. El coeficiente de variación fue $4.55 \%$. 
En la segunda, el análisis de varianza para el tramo medio poda, no presentó diferencia estadística significativa entre tratamientos. En promedio el tramo medio alto fue 4.13. El coeficiente de variación fue $7.00 \%$.

El tramo medio bajo presentó valores de 3.71 y 3.78 en la primera y segunda lectura respectivamente; el tramo medio alto presentó una media de 4.75 en la primera lectura y 4.13 en la segunda, lo que sumado nos da un valor de 8.46 que corresponde a un fuste recto en la primera lectura y 7.91 en la segunda lectura correspondiente a un fuste con curva leve, según los dicho en el cuadro 4 de escala para valoración de la rectitud del fuste propuesta por HERRERA, B. 2013.

El testigo con un valor de 7.93 presenta una curva leve mientras que los tratamientos con poda al $50 \%$ y $70 \%$ presentan valores de 8.41 y 8.43 respectivamente, lo cual corresponde a un fuste recto, motivo por el cual podríamos decir que la poda si influye en la rectitud del fuste.

\section{Número de ramas podadas}

En la primera poda el análisis de varianza para el número de ramas cortadas, presentó diferencia estadística altamente significativa entre tratamientos. En promedio el número de ramas cortadas en la primera poda fue 3.90. El coeficiente de variación fue $23.81 \%$.

En la prueba de Tukey al 5\% para el número de ramas cortadas en la primera poda, presentó 2 rangos; 1 poda 50, 60 y $70 \%$ se ubicaron en el rango "A" con valores de 4.03, 5.95 y 5.63 ramas podadas cada una de ellas respectivamente, mientras que el testigo (T0) se ubicó en el rango "B" con un valor de 0.00 ramas.

La poda realizada al $60 \%$ tiene mayor número de ramas que la realizada en un $70 \%$, esto se debe a la separación entre verticilos la cual fue más distante en los árboles con poda al $70 \%$.

En la segunda poda, el análisis de varianza para el número de ramas cortadas, presentó diferencia estadística altamente significativa entre tratamientos. En promedio el número de ramas cortadas en la segunda poda fue 7.70. El coeficiente de variación fue $17.44 \%$.

En la prueba de Tukey al 5\% para el número de ramas cortadas en la segunda poda, presentó 4 rangos; la poda $70 \%$ (T3) se ubicó en el rango "A" con un valor de 11.66 ramas podadas, mientras que el testigo (T0) se ubicó en el rango "C" con un valor de 0.00 ramas, los otros tratamientos se ubicaron en un rango intermedio.

El número de ramas podadas va en aumento de acuerdo a lo establecido para cada tratamiento del ensayo. 


\section{Bifurcación}

La mayor bifurcación se presentó en los árboles de la poda al $50 \%$ (T1) con un valor de 3 árboles seguido de los otros tratamiento con 2 árboles bifurcados cada uno de ellos respectivamente.

ALGUACIL, (2006), manifiesta que la bifurcación corresponde a la altura del tallo o fuste en la cual se diferencia un eje principal y es una característica con alto valor económico, ya que puede limitar la producción de madera comercial, los resultados obtenidos en la investigación muestra que no existe mayor riesgo de pérdida en la producción de madera.

El mayor número de árboles bifurcados que presentó el ensayo fue en la poda al $50 \%$ lo cual se debe posiblemente a caracteres hereditarios de la especie o a algún daño mecánico que pudo sufrir la planta ya sea este producido por aves, insectos o el viento.

\section{Ramas desgajadas}

El mayor problema de desgaje se ha presentado en el testigo (T0) con un valor de 9 árboles mientras que el tratamiento de poda al 70 \% (T3) no presentó esto este problema.

El mayor número de ramas desgajadas presentó el testigo (T0), lo cual se debe a que al no ser podados estos árboles las ramas se alargan, aumentan en diámetro y por lo tanto adquieren mayor peso produciéndose el desgaje de las mismas.

\section{Determinación de la intensidad de poda más adecuada}

La intensidad de poda tiene una gran influencia en el diámetro de la especie ya que el testigo presenta valores altos en la evaluación, lo cual se debe a que estos árboles no sufrieron ningún estrés al no ser podados por tal motivo su crecimiento no mermó a ningún momento. Las especies no podadas tienen valores variantes entre $2.50,4.44$ y $6.39 \mathrm{~cm}$ en la primera, segunda y tercera medición respectivamente que se realizó con pie de rey; así mismo la cuarta, quinta y sexta evaluación que se midió utilizando la forcípula alcanzó valores máximos de $3.27,4.73$ y $5.98 \mathrm{~cm}$ respectivamente. En cuanto a la intensidad de poda podemos decir que la realizada al $60 \%$ fue la que presentó mejor diámetro durante el desarrollo del ensayo con valores de 2.26, 3.50 y 5.43 en la primera, segunda y tercera lectura realizadas con el pie de rey; mientras que la cuarta, quinta y sexta medición realizada con la forcípula alcanzó valores de diámetro correspondientes a 3.66, 4.67 y 5.76 respectivamente 


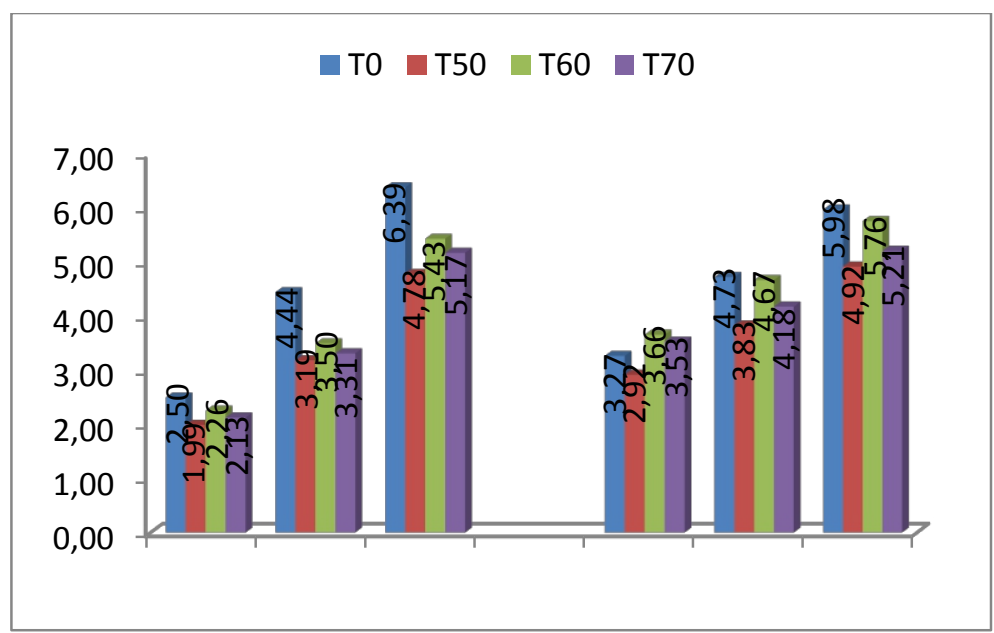

Grafico 1. Valores de diámetro en los diferentes tratamientos

En relación a la rectitud del fuste las podas al 50 y $70 \%$ presentaron un fuste recto con valores promedio de 8.60 en la primera lectura y 8,23 en la segunda lectura para la poda al $50 \%$; mientras que la poda al $70 \%$ presentó valores de 8.49 y 8.37 en la primera y segunda lectura respectivamente lo que corresponde a un fuste recto según el cuadro de interpretación propuesto por HERRERA, V (2013); la poda al 60\% y el testigo en la primera lectura presentan valores de 8.38 y 8.36 respectivamente que corresponde a un fuste recto y en la segunda lectura presentan valores decrecientes de 7.58 y 7.49 cada uno de ellos respectivamente lo que corresponde a una curva leve.

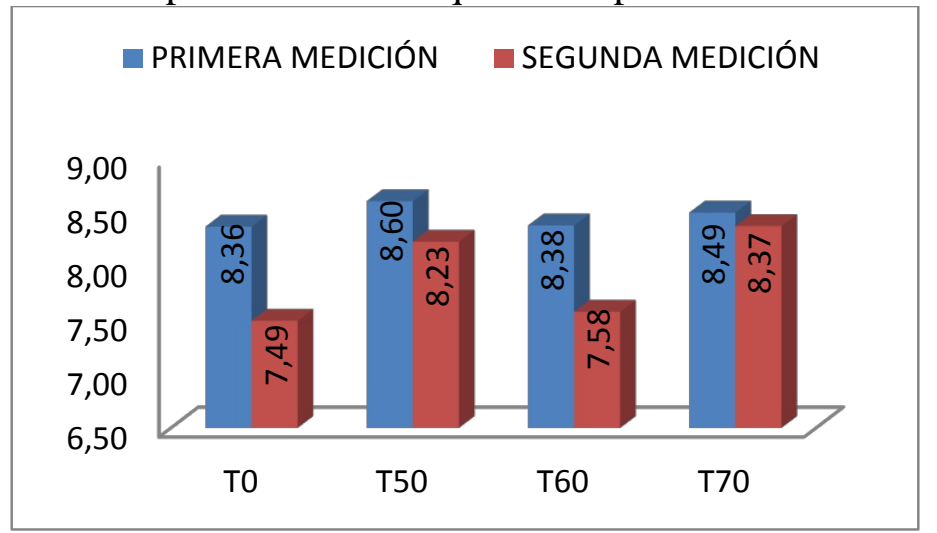

Gráfico 2. Valores de la forma del fuste en los diferentes tratamientos

\section{Conclusiones}

Los mejores incrementos en diámetros fueron alcanzados por el testigo. Con la poda del $60 \%$ se obtienen los mejores diámetros, luego del testigo, pero la forma del fuste del árbol presenta una curva leve con relación a la intensidad de poda del $50 \%$ y $70 \%$ donde los árboles son más rectos pero con diámetros menores. 
Las podas de formación no influyen en el crecimiento en altura de la planta, pero si en el incremento del diámetro. La falta de poda en melina ocasiona el desgaje de las ramas provocando daño al fuste del árbol.

\section{References:}

1. AGROSOT Ltda. 2000. G. arbórea Roxb. Trees versión 2. SerieEspecial Forestales. Reporte de especies No. 4. Medellín, Colombia. pp. 2-9. (Internet). Recuperado de: htt//www.colforest.com.co/serie_especies_forestales/gmelinaarborea.pdf.

2.ALGUACIL, F. 2006 Árboles singulares - Medio ambiente; Ingeniero de Montes Recuperado de: http://www.elpadul.es/medio/arbolsin.htmAHUMADA, P., 1965 Recuperado de: http://es.wikipedia.org/wiki/Evaluaci\%C3\%B3nCHAVARRÍA y VALERIO., 1993 "Manual de Técnicas de Desarrollo de Gmelina arbórea". Recuperado de: www.text.manual//melina.pdf

3.COMISIÓN NACIONAL FORESTAL. (CONAFOR). Gmelina arbórea Roxb.

Recuperado de:http:/www.conafor.gob.mx/programás_nacionales_forestales/pron are/fichas\%20tecnicas/Gmelina\%20arborea.pdf. (Consultada: $28 \mathrm{de}$ noviembre 2012).

4.FAO. 2002. Evaluación de los recursos forestales mundiales 2000. Informe

5.GEILFUS, F. 2010. El árbol al servicio del agricultor: Principios y técnicas. de:http://books.google.com.ec/books?id=eCQOAQAAIAAJ\&pg=PA $511 \& \mathrm{lpg}=\mathrm{PA} 511 \& \mathrm{dq}=$ poda + de+formaci $\% \mathrm{C} 3 \% \mathrm{~B} 3 \mathrm{n}+\mathrm{en}+$ maderables

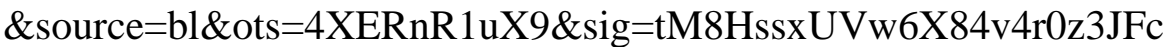
jUg\&hl=es\&sa=X\&ei=3e27UL_fGoq8ATFtIGoDQ\&ved=0CDYQ6 AEwAg\#v=onepage $\& q=$ poda $\% 20 \mathrm{de} \% 20$ formaci $\% \mathrm{C} 3 \% \mathrm{~B} 3 \mathrm{n} \% 20 \mathrm{en} \%$ 20maderables $\& \mathrm{f}=$ false

6.HERRERA, B. 2013. Ing. Forestal. SERAGROFOREST S.A. Información personal.

7.LAMB, A.F.A. 1968. Gmelina arbórea In: Fast Growing Timber Trees of Lowland Tropics. No. 1. University of Oxford, departament of forestry. Comm. For. Inst. 34p. 
8.MURILLO, O. 1991. Metodología para el control de la calidad en plantaciones forestales. Cómo estimar la calidad de la materia prima desde el árbol en pie? Instituto Tecnológico de Costa Rica. Recuperado

de:

http://www.una.ac.cr/inis/docs/teca/temas/olmamur.pdf

9.OBREGÓN, C. 2005. Gmelina arbórea Versatilidad, Recuperado de y Productividad Sostenible para el Futuro Bogotá - Colombia $20 \mathrm{p}$

10. OHLAND, C. 2000. Recomendaciones para el manejo de Gmelina arbórea. Costa Rica. MINAE-ITCR-COSEFORMA-CCF-GTZ. 53 p.

11. Organización de las Naciones Unidas para la Agricultura y la Alimentación. (FAO) 2002. Plantaciones forestales. (Internet). Recuperado de: http://www.fao.org/documents/show_cdr.asp?url_file=/docrep/005/y 1997s/y1997s09htm (Consultada: 25 de octubre del 2012) para la Agricultura y la Alimentación Roma, 468 p.

12. PATIÑO, V. F., O. Cedeños, S., V. M. Juárez, G. y R. Bertoni, V. 1982. Gmelina arbórea Roxb. Una especie promisoria en el trópico mexicano. SARH, INIF, CIFTROH. Vol. Téc. No. 3. Campeche, Campeche, México. 43 p., principal. Estudio FAO Montes 140. Organización de las Naciones Unidas

13. PEREZ, J (1995) Disponible http://www.ite.educacion.es/formacion/materiales/90/cd_09/cursofor/ cap_4/cap4a.htm

14. ROJAS, R. F., D. Arias, A., R. Moya, R., A. Meza, M., O. Murillo, G. y M. Arguedas, G. 2004. Manual para productores de melina Gmelina arbórea en Costa Rica. Recuperado de: http://www.fonafifo.go.cr/text_files/proyectos/Manual\%20 Prod\%20Melina.pdf

15. SANCHEZ, J

(1988) Disponible http://www.definicion.org/evaluacion

16. STRUCK, G. and Dohrenbusch, A. 2000. Development of a new branchiness index ASIX - A simple tool to describe branchiness in young deciduous forest stands. Ann. For. Sci.57, 811-818.

17. TRUJILlO, E. 2007. Guía de Reforestación. Bogotá. 280 p. Recuperado de: http://www.revista- 
mm.com/ediciones/rev75/forestal_poda.pdf.(Consultada: 25 de octubre del 2012)

18. TORRES, J.M. y Magaña, T.O. 2001. Evaluación de plantaciones forestales. Limusa

19. VALLEJO, 1996. Evaluación del crecimiento y calidad de plantación en Gmelina arbórea ROXB.

20. ZOBEL, B. J. y J. T. Talbert. 1988. Técnicas de mejoramiento genético de árboles forestales. México. Ed. Limusa. pp 96-138 